\title{
A Final Price Prediction Model for online English Auctions-A Neuro Fuzzy Approach
}

\author{
Chin-Shien Lin ${ }^{1}$, Shih -Yu Chou ${ }^{2}$, Chi-Hong Chen ${ }^{3}$, Tai-Ru Ho ${ }^{3}$, Yu-Chen $\mathrm{Hsieh}^{4}$ \\ ${ }^{1}$ :Professor, Department of Business Administration, National Chung Hsing University,E-mail: csvincentlin@dragon.nchu.edu.tw \\ ${ }^{2}$ :Professor, Department of Marketing, National Chung Hsing University,E-mail: sychou@dragon.nchu.edu.tw \\ ${ }^{3}$ :Graduate Student, Institute of Electronic Commerce, National Chung Hsing University \\ ${ }^{4}$ :Graduate Student, Educational Measurement and Statistics, National Taichung Teachers College
}

\begin{abstract}
Markov Chain Model provides a concise mathematical model to describe the online English auction process, converting the complicated interaction between the bidders and auctioneer into a tractable mathematical problem, which is a milestone for researches involved in this area. However, the assumptions about the parameters are not consistent with the actual phenomena, for example, the distribution of the private values and the arrival rates. Furthermore it is hard to obtain the values of these parameters. In this paper, a hybrid method, neuro fuzzy, is proposed to predict the final price in addition to exploring the complicated, possibly nonlinear, relationship among the auction mechanisms and final price. The empirical results show that neuro fuzzy system can predict the final price accurately much better than the others, which is of great help for the buyers to avoid overpricing and for the sellers to facilitate the auction. Besides, the knowledge base obtained from neuro fuzzy provides the elaborative relationship among the variables, which can be further tested for theory building.
\end{abstract}

\section{Introduction}

Auctions have long been of special interest to economists due to their explicit mechanisms to describe how prices are formed (Hendricks and Paarsch, 1995). However, most of the issues are mainly focused on the design mechanism from either the sellers or buyers' point of view or from both. There have been few researches done in determining the final prices. Final price prediction for online auctions, which involves the modeling of uncertainty regarding the bidding process, is a challenging task primarily due to the variety of factors changing in auction settings (Schapire et al., 2002). Even if all the factors were accounted for, there still exists the uncertainty in human behavior when bidding in auctions. The relationship among the final price and the related factors can be more than just a linear one. Nevertheless, this issue is related to the utility of bidders, revenue of sellers, and allocation efficiency from point of view of whole social welfare. More and more researchers investigate this issue.

Markov Chain Model provides a concise mathematical model to describe the online English auction process, converting the complicated interaction between the bidders and auctioneer into a tractable mathematical problem, which is a milestone for researches involved in this area. However, the assumptions about the parameters are not consistent with the actual phenomena, for example, the distribution of the private values and the arrival rates. Furthermore it is hard to obtain the values of these parameters.

On the other hand, as far as the nonlinear problem is concerned, the progress in artificial intelligence technology now provides a possible alternative that deserves further exploration in solving this problem. Among the available methods, expert system, fuzzy logic, and neural network are three of the most commonly used in helping managers make decisions. The expert system can embed the past experience into the system; fuzzy logic can describe the problem in a way that is close to the human reasoning process and accommodate the inaccuracy and uncertainty associated with the data; the neural network can learn from historical data. However, the difficulty with the acquisition of the knowledge base for both the expert system and fuzzy logic, and the difficulty with the causal explanation through the construction of appropriate 'real' relations among the variables for the neural network model have constrained the application of these three methods. A method which can combine the advantages of these three methods while avoiding their disadvantages would seem to hold some promise.

Since predicting the final price of the auction can be of great help for bidders to set a reference price to avoid overpricing and for sellers to set the auction rules to facilitate the completion of the auction (Greenleaf, 2004), this paper is trying to apply neuro fuzzy technique to model the price prediction problem. This proposed system can do the fuzzy reasoning and, through learning, can adjust the relative importance of each fuzzy rule. Furthermore, the knowledge base obtained from this technique can be used as 
an explanation about how the price is affected by the factors, facilitating the understanding about the auctions. Four benchmark models, regression, neural network, Markov chain model, and classical expected value are constructed for comparison to show the validation of the proposed model. The rest of this paper is structured as follows. Section 2 briefly provides an overview of online auction literature. Section 3 describes how a neuro fuzzy system is constructed. The research methodology is described in Section 4. Empirical results are shown in Section 5 and finally some conclusions are given in section 6 .

\section{Literature Review}

There are four basic types of auctions when a single item is to be sold, the ascending bid auction (also called the open or English auction), the descending bid auction (also called Dutch auction), the first-price sealed-bid auction, and the second-price sealed-bid auction (also called Vickrey auction) (Klemperer, 1999; McAfee and McMillan, 1987). Among them English auction is the most well-known and most widely used one. Since the English auction is the most commonly used format of auction on the internet, this paper is aiming to explore the relationship among the main attributes and the final price for the English auctions.

With the great success of online auction, there already exists a vast body of researchers from economics, finance, and e-commerce who studied the general theory of auction and attempted to test presented theories in real-world environments. (McAfee and McMillan, 1987 ;

Hendricks and Paarsch, 1995 ; Kauffman and Wood ,2004 ; Kamins et al., 2004 ; Vakrat and Seidmann,2000 ; Bapna, Goes and Gupta, 2003). The types of those researches can divide into three parts, design of the mechanism, the final price prediction, and the design of automated bidding agent. Since there is a lack in the final price prediction, and English auction is one of the most popular auction types, this research will focus on the final price prediction of the on line English auction.

\section{The Construction of a Neuro Fuzzy System}

Fuzzy logic utilizes fuzzy sets defined by membership functions in logical expressions to deal with the extent to which the object belongs to the set. The membership function $\mu_{A}(x)$ with a value varying between 0 and 1 denotes the degree of membership to which object $x$ belongs to fuzzy set $A$. The closer this value is to 1 , the higher the membership of $x$ belonging to set $A$. A fuzzy logic system is constructed by introducing the logical relation of the "IF-THEN" rules to express the relationship among independent and dependent variables. For the clarity of the explanation, we only take two independent variables and one dependent variable for example. Let $\mathrm{P}_{\mathrm{s}}, \mathrm{P}_{\mathrm{m}}$, and $\mathrm{P}_{\mathrm{f}}$ represent the starting price, external reference price, and final price of an auction, respectively.

A fuzzy logic rule is stated as follows:

IF $P_{s}$ is low, and $P_{m}$ is medium, then $P_{f}$ is medium.

where $\mathrm{P}_{\mathrm{s}}, \mathrm{P}_{\mathrm{m}}$, and $\mathrm{P}_{\mathrm{f}}$ are called linguisic variables and high, medium, and low are called linguistic terms. The construction of a fuzzy logic system consists of three major steps: fuzzification, construction of knowledge base, and defuzzification.

Basically neuro fuzzy system is a fuzzy logic system with a learning algorithm derived from or inspired by neural network theory to determine its parameters, including the parameters of the membership function and the relative importance of each fuzzy rule (Buckley and Hayashi, 1995). The most common approach to be used to combine these two techniques is so-called Fuzzy Associative Memory (FAM) proposed by Kosko (1992) and this paper adopts this approach. A FAM attempts to use neural networks to implement the desired mapping for fuzzy systems by applying fuzzy rules to a set of inputs, combining the consequents of each rule, and producing a value for the output variable. Each rule is associated with a weight factor that represents the importance of the rule in relevance to the other rules in the system. The errors between the results computed by the FAM system and the desired output are used to modify the weights. The training process will stop until the error is less than a certain threshold value. A fuzzy logic system is constructed by using the complete knowledge base to state the relationship among independent and dependent variables. Then the knowledge base is fine tuned by using the learning ability of neural network based on the training data set. Finally we use the testing data set to validate the obtained model.

\section{Data Collection}

The data set of this study was collected from the real world online auction website, Yahoo-Kimo Auction, by a spider program written in Java. Digital camera product is chosen as the research target due to its variety of goods and prices. The spider program visited the Yahoo-Kimo Auction home page first, and then obtained the link to Digital camera auction page, collecting the IDs of all Digital camera auctions closed in pervious months. Each auction ID was used to construct a Web URL. After that all the details about each auction can be retrieved. 
Totally 110 digital camera auctions are collected with due date from December 3, 2004 to January 20,2005 . The data is divided into two sets, training data set and testing data set. The training data set, used for model construction, consisted of 73 examples (66\%) and the testing set, used for validation, consisted of 37 examples (34\%). To show the robustness of the cross validation, we randomly divide the data into two parts for ten times, and do the model construction and prediction for each division. Mean squared errors (MSE) is used as the performance criterion, which is calculated as MSE $=\sum_{i=1}^{n}\left(y_{i}-\hat{y}_{i}\right)^{2} / n$, where $\quad y_{i}$ and $\hat{y}_{i}$ represents the actual value and predicted value of auction $i$ respectively, and $\mathrm{n}$ is the number of auctions.

\section{Empirical Results}

Table 1 shows the pair-wise comparison among five methods. Each cell shows the t-value of the difference of MSE between the method at the row position and the method at the column position, and the figure in the parenthesis is the corresponding $\mathrm{p}$-value of the difference(ex, -0.338 indicates that the MSE of neural network is less than that of regression, but not significant). The empirical results indicate that neruo fuzzy performs the best among these five methods in testing data set with significance level set at 0.01 . Neural network performs better than regression significantly on the training data sets, but not significantly on testing data set. Table 2 lists the rules obtained from the first training data set with relative importance (Degree of Support, Dos) greater than 0.9. Take rule 4 for example, "IF Participation is high, Price_related is high, Product_info is medium, and Seller_ratings is high, THEN $\mathrm{P}_{\mathrm{f}}$ is very high." This statement does follow what we expect. Similarly, according to rule 1, "IF Participation is low, Price_related is medium, Product_info is less, and Seller_ratings is low, THEN $\mathrm{P}_{\mathrm{f}}^{-}$is low", this statement also conforms to our intuition. As for rule 2 and rule 3, they show how the complicated interactions among the variables work, which can facilitate the understanding of the impacts on the final price of these related variables.

\section{Conclusions and Applications}

This paper is trying to propose a final price prediction model for English auctions by using neuro fuzzy technique to catch the complicated relationship, especially the nonlinear one, among the key factors involved in an auction. The empirical results show that neuro fuzzy performs the best no matter in the training data sets or testing data sets in terms of the forecasting accuracy. Besides, the knowledge base obtained from the neuro fuzzy system can not only provide practitioners some insights in understanding the bidding process but also provide the academia the basis for constructing hypotheses for further investigation. This proposed neuro fuzzy system has shown a promising perspective to explore the online auction.

This proposed model can be of great help for both the bidders and sellers. With the help of this proposed model to predict the closing prices accurately, the bidders can determine which auction is close to his/her willingness-to-pay price to place a bid, avoiding the overpricing and reducing the uncertainty in winning the item in the auction. On the other hand, the model can also be used to provide sellers the suggestions about the auction rules to facilitate the completion of the auction and to maximize the final price. Besides, this price prediction model can also facilitate the allocation efficiency from the market point of view. In other words, through this model bidders and sellers can achieve the final price in a more effective way with the maximum utility satisfaction in both sides.

The main contribution of this study is to construct an effective and accurate final-price model by using neuro fuzzy based on the related attributes of the auction. In this proposed model, external reference price plays an important role in determining the final price. However for those auctions that the external reference prices do not exist (such as antiques, art, and collectibles etc.) this model needs to be modified.

This paper aims to explore the complicated (nonlinear) relationship among the variables by using neuro fuzzy. The empirical results show the promising possibility to explore the nonlinear relationship. This research can be further pursued by extending to different product type and even including some other related variables not explored in this research.

\section{References}

[1] Bapna, R., Goes, P and Gupta, A., "Replicating Online Yankee Auctions to Analyze Auctioneers' and Bidders' Strategies," Information Systems Research, Vol. 14 No. 3, pp. 244-268, 2003.

[2] Buckley, J. J., and Hayashi, Y., "Neural networks for fuzzy systems, Fuzzy Sets and Systems," 71, 1995, pp. 265-276.

[3] Greenleaf, E. A., "Reserves, Regret, and Rejoicing in Open English Auctions," Journal of Consumer Research, 31, 2, 2004, pp. 264-273. 
[4] Hendricks, K., and Paarsch, H. J., “A survey of recent empirical work concerning auctions," The Canadian Journal of Economics, 28, 2, 1995, pp. 403-426.

[5] Kamins, M. A., Dreze, X., and Folkes, V. S.,"Effects of Seller-Supplied Prices on Buyers' Product Evaluations: Reference Prices in an Internet Auction Context," Journal of Consumer Research, 30, 4, 2004, pp. 622-628.

[6] Kauffman, R. J., and Wood, C. A., "Doing Their Bidding: An Empirical Examination of Factors That Affect a Buyer's Utility in Internet Auctions," Forthcoming in Information Technology and Management, 2004.

[7] Klemperer, P., "Auction Theory: A Guide to the Literature," Journal of Economic Surveys, 13, 3, 1999, pp. 227-286.

[8] Kosko, B., Neural networks and fuzzy system: A Dynamic System Approach to Machine Intelligence, Englewood Cliffs, N.J., Prentice Hall, 1992.

[9] McAfee, R. P., and McMillan, J., "Auctions and Bidding", Journal of Economic Literature, 25, 1987, pp. 699-738.

[10] Schapire, R. E., Stone, P., McAllester, D., Littman, M. L., and Csirik, J. A.,

"Modeling auction price uncertainty using boosting-based conditional density estimation," In Nineteenth International Conference on Machine Learning, Sydney, 2002.

[11] Vakrat, Y., and Seidmann, A., "Implications of the Bidders' Arrival Process on the Design of Online Auctions," Proceedings of the 33rd Hawaii International Conference on System Sciences 6, 2000, pp. 1-10.

Table 1.Pairwise Comparisons of MSE for testing data set

\begin{tabular}{|c|c|c|c|c|c|}
\hline Method & Regression & $\mathrm{NN}$ & NF & $\mathrm{MC}$ & Classical \\
\hline \multirow{2}{*}{ Regression } & & -0.338 & $7.049 * *$ & $-12.552 * *$ & $-12.780 * *$ \\
\hline & & $(0.743)$ & $(0.000)$ & $(0.000)$ & $(0.000)$ \\
\hline \multirow{2}{*}{$\mathrm{NN}^{* *}$} & -0.338 & & $8.125^{* *}$ & $-13.003 * *$ & $-12.827 * *$ \\
\hline & $(0.743)$ & & $(0.000)$ & $(0.000)$ & $(0.000)$ \\
\hline \multirow{2}{*}{$\mathrm{NF}^{* * *}$} & $7.049 * *$ & $8.125 * *$ & & $-19.145 * *$ & $-13.953 * *$ \\
\hline & $(0.000)$ & $(0.000)$ & & $(0.000)$ & $(0.000)$ \\
\hline \multirow{2}{*}{$\mathrm{MC}^{* *}$} & $-12.552 * *$ & $-13.003 * *$ & $-19.145^{* *}$ & & $-4.773 * *$ \\
\hline & $(0.000)$ & $(0.000)$ & $(0.000)$ & & $(0.001)$ \\
\hline \multirow{2}{*}{ Classical** } & $-12.780 * *$ & $-12.827 * *$ & $-13.953 * *$ & $-4.773 * *$ & \\
\hline & $(0.000)$ & $(0.000)$ & $(0.000)$ & $(0.001)$ & \\
\hline
\end{tabular}

t-value, (p-value) $* *$ : significance level at 0.01

Table 2. Knowledge base with relative importance greater than 0.9

\begin{tabular}{|l|c|c|c|c|c|c|}
\hline \multicolumn{7}{|c|}{ Rule Base } \\
\hline No & Participation & Price_related & Product_info & Seller \\
Reputation & DoS & THEN \\
\hline 1 & low & medium & less & low & 1.00 & low \\
\hline 2 & high & medium & more & high & 0.92 & medium \\
\hline 3 & medium & high & more & high & 0.91 & very high \\
\hline 4 & high & high & medium & high & 0.91 & very high \\
\hline
\end{tabular}

